\title{
Balkanologie
}

Balkanologie Revue d'études pluridisciplinaires

Vol. VII, $n^{\circ} 1$ | 2003

Volume VII Numéro 1

\section{Les albanais en Grèce}

Le rôle des réseaux préexistants

\section{Pierre Sintès}

\section{OpenEdition}

\section{Journals}

Édition électronique

URL : http://journals.openedition.org/balkanologie/474

DOI : $10.4000 /$ balkanologie.474

ISSN : 1965-0582

Éditeur

Association française d'études sur les Balkans (Afebalk)

\section{Édition imprimée}

Date de publication : 1 juin 2003

Pagination : 111-133

ISSN : 1279-7952

Référence électronique

Pierre Sintès, «Les albanais en Grèce », Balkanologie [En ligne], Vol. VII, $n^{\circ} 1$ | 2003, mis en ligne le 18 février 2009, consulté le 17 décembre 2020. URL : http://journals.openedition.org/balkanologie/474 ; DOI : https://doi.org/10.4000/balkanologie.474 


\title{
LES ALBANAIS EN GRÈCE LE RÔLE DES RÉSEAUX PRÉEXISTANTS
}

\author{
Pierre Sintès*
}

Depuis le début des années 1990, la migration des travailleurs albanais vers la Grèce est l'un des phénomènes démographiques majeurs touchant le sud de la péninsule balkanique. Ce flux concernerait en effet un groupe de plus de 500000 personnes selon les estimations fréquemment prononcées à Athènes. Les Albanais constitueraient plus de la moitié des étrangers présents sur le sol grec et sont à l'origine de la véritable transformation de ce pays en l'une des premières terres d'accueil de l'Union européenne proportionnellement à sa population (part estimée à $10 \%$ ).

Les principales originalités de cette migration sont sa grande rapidité et sa diffusion spatiale. On retrouve en effet des Albanais sur l'ensemble du territoire grec, jusqu'aux villages les plus isolés des montagnes où des îles de la mer Egée $^{1}$. En fonction de quoi se décide le lieu de destination des migrants ${ }^{2}$ ? Si les explications les plus simples sont apparues, de prime abord, satisfaisantes (répartition en fonction des grandes zones d'emploi du pays, importance des axes

\footnotetext{
• Ecole Française d'Athènes, Centre de la Méditerranée Moderne et Contemporaine, Université de Nice Sophia-Antipolis.
}

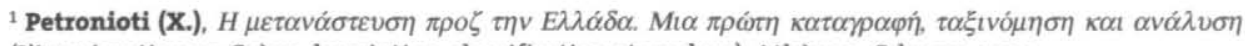
(L'immigration en Grèce, description, classification et analyse), Athènes : Odyssea, 1993.

\footnotetext{
${ }^{2}$ Lorsque, vivant à Buenos Aires, je commençais à apprendre le Grec moderne, je suivais les cours d'une jeune grecque de bonne famille, épouse d'un français au sang bleu et, elle mème descendante d'une famille ayant géré au XIXe siècle, pour le compte des Ottomans, ce qui allait devenir la Roumanie. Aux premières évocations des migrations qui touchaient aujourd'hui la Grèce en provenance des pays de l'Est, elle me raconta l'histoire de ces jeunes Roumains venus frapper à la porte de sa maison familiale l'année précédente pour demander assistance. Ils avaient obtenu son nom de leur grand-mère et étaient à la recherche d'une première base pour s'établir en Grèce. Quelques années après, quand j'ai entamé mes recherches de doctorat sur la migration des Albanais en Grèce, j'ai gardé en mémoire cette anecdote pour en faire une hypothèse de travail et orienter mes recherches vers ces réseaux migratoires qui mobilisent des connexions anciennes liant la Grèce et les autres pays des Balkans.
} 
de communication...), les récits de vie recueillis lors d'enquêtes répétées en Albanie et en Grèce m'ont permis de constater que d'autres logiques seraient à l'œuvre pour orienter les pas des migrants, notamment celles qui font appel à des réseaux anciens, tissés durant les périodes précédant la dictature d'Enver Hoxha.

Cette constatation est à l'origine de mon intérêt pour le fonctionnement migratoire de certaines communautés présentant des liens historiques avec la Grèce. Plus particulièrement, le cas des communautés transfrontalières donne l'occasion d'illustrer l'influence de ces réseaux originaux. À travers cet exemple, c'est la prégnance du lien familial dans la structuration de la migration qui apparaît, mais aussi la manière dont certaines solidarités ont pu être ravivées par l'ouverture récente de la frontière et exploitées, au moins un temps, dans le projet migratoire. La présente étude a pour but de décrire et d'expliquer l'utilisation de ce type de réseau et les pratiques spatiales qui lui sont associées. Il s'agit aussi de montrer que ce phénomène s'inscrit dans un ensemble de processus qui orientent les choix du migrant ; il peut en être même, dans certains cas, la procédure dominante.

\section{LES FACTEURS DE LA RÉPARTITION DES ALBANAIS EN GRÈCE}

Pour expliquer la répartition des immigrés dans leur pays d'accueil, on peut faire appel aux logiques classiques considérant la migration comme une compensation de l'inégale répartition spatiale des richesses et du travail ${ }^{3}$. Une telle lecture s'inscrit dans la droite ligne des travaux qui, depuis la seconde moitié du XIX ${ }^{\mathrm{e}}$ siècle, envisagent les mobilités comme la simple expression des forces d'attraction ou de répulsion exercées par les espaces concernés4. Le déplacement correspondrait ainsi à la fuite logique depuis les espaces pauvres et surpeuplés vers les zones riches et attractives. Concernant les mouvements de population dans les Balkans, c'est le schéma explicatif utilisé par Jovan Cvijić quand celui-ci oppose les "pays ruches" d'où partent les mouvements dits "métanastasiques", à des espaces attractifs où s'installent les nouveaux venus 5 .

\footnotetext{
3 Escallier (Robert), " Populations et systèmes migratoires du monde arabe ”, in Troi (Jean-François), Maghreb - Moyen Orient, mutations, Paris : SEDES, 1995.

4 Il s'agit de la théorie du push and pull ou " modèle gravitaire " suggérée à la fin du XIXe siècle par E. G. Ravenstein et formulée par la suite par R. D. Mac Enzie (cité par Thumerelle (Pierre-Jean), Peuples en mouvement, la mobilité spatiale des populations, Paris : SEDES, 1986, p. 95). Elle postule que les mouvements de population procèdent de phénomènes d'attraction et de répulsion. Cette approche explique les mobilités spatiales à une échelle macro et font des migrations le résultat de différences de potentiel économique entre les zones concernées par le mouvement.
}

5 Cvijić (Jovan), “ Les mouvements métanastasiques de la péninsule balkanique ”, Monde Slave, (1), 1917. 
Pourtant, si cette vision des choses permet d'expliquer à grands traits la répartition des populations, elle demeure mécaniste ${ }^{6}$ et ne demande qu'à être contredite par des études plus précises.

\section{Les limites du push and pull}

La répartition des Albanais en Grèce peut être présentée grâce à la cartographie de certaines sources statistiques. Les plus récentes et les plus fiables seraient celles du dernier recensement de la population grecque de 2001 . Malheureusement, les résultats ne sont pas encore accessibles. C'est pourquoi nous devrons nous contenter, malgré leurs imperfections, des statistiques concernant les demandes de cartes vertes 7 effectuées en 1998 auprès de $l^{\prime}$ Office du travail et de la main d'œuvre ${ }^{8}$. Ces données offrent de nombreuses lacunes (par exemple l'exclusion des Albanais ayant pu faire valoir, à tort ou à raison, une origine grecque ou encore, l'absence évidente de ceux qui, dans les conditions les plus précaires, ont été contraints à la clandestinité) mais elles constituent pour l'heure l'unique source disponible.

\section{UN CALQUE DE LA GÉOGRAPHIE ÉCONOMIQUE}

La géographie économique de la Grèce semble être déterminante dans la répartition des Albanais qui apparait comme un simple calque de la carte de l'emploi en Grèce. A cette échelle, le classique push and pull est opératoire. En effet, les Albanais se répartissent en très grande majorité le long des axes de dynamisme de l'économie grecque c'est à dire (voir document $n^{\circ}{ }^{\circ}$ ) le long de la route Patras-Athènes-Thessalonique. Mais on peut en arriver à d'autres conclusions si l'on introduit, pour ce résultat, une correction tenant compte de la population région par région. En effet, en calculant de la sorte le rapport de la population albanaise immigrée ayant fait une demande de carte verte à la population totale en 2001 , on obtient un résultat bien différent du précédent et l'importance des axes de développement s'estompe au profit d'autres zones (régions agricoles du pourtour du Péloponnèse, Béotie et Thessalie, zones frontalières de l'Albanie et îles touristiques). Un tel résultat nous amène à conclure que les Albanais sont répartis sur l'ensemble du territoire grec et que leur poids "relatif" est même, sans doute, plus grand dans les zones rurales qu'urbaines 9 .

\footnotetext{
${ }^{6}$ Pour l'usage du vocabulaire relatif à la mécanique des fluides pour désigner les migrations, voir Green (Nancy), Repenser les migrations, Paris : PUF / Le ncud gordien, 2002.

7 Les cartes vertes sont des permis de travail et de séjour créés à partir de 1997 par décret présidentiel. Elles étaient destinées à la régularisation mais ont été très longtemps l'unique titre de séjour utilisé par les étrangers extra-communautaires.
}

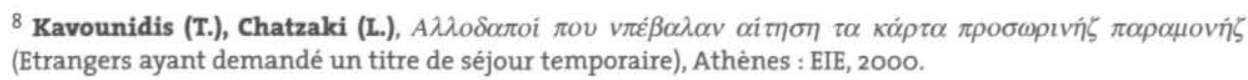

9 Sintès (Pierre), “ Immigration, réseaux et espace métropolitain, le cas athénien ", Cahiers de la Méditerranée (Les), (63), 2002. 


\section{LES FACTEURS DE LA RÉPARTITION : RÉSEAUX ET MIGRATION À LA CHAÎNE}

À quoi peut-on attribuer cette diffusion ? Certes, les caractéristiques du marché du travail agricole offre des conditions favorables pour l'emploi des immigrés mais, aussi, des séjours de recherches dans la plaine de Corinthe (région de la Voha) ont permis de mettre en évidence de manière claire l'importance de réseaux - essentiellement familiaux - liant un point du territoire albanais au départ et un autre point en territoire grec à l'arrivée. Les Albanais résidant ou travaillant, par exemple, dans le village de Vohaïko sont venus exclusivement des villages de Hajmel (région de Shkodër), de ceux de la région de Peshkopi et de Karjan (région de Gjirokastër). Des informations obtenues auprès de la municipalité de la Voha, à l'occasion de la seconde campagne de régularisation de 2000 , ont permis de constater qu'en moyenne, $80 \%$ des Albanais de même origine résident dans le même village. Ce phénomène de "migration à la chaîne" donne un grand poids au choix des pionniers ; à leur suite viendront s'installer les suivants. Ainsi, dans le cas de Vohaiiko, ce sont près de 40 personnes qui sont venues depuis la région de Gjirokastër après l'installation du premier en 1991. Les récits de ces pionniers dans la Voha font souvent intervenir le hasard voire la volonté divine pour expliquer leur établissement dans la plaine de Corinthe. Pourtant, après avoir passé plus de temps avec les différentes personnes rencontrées, de nouvelles informations finissent par être données. Certains ne seraient pas venus au hasard dans la région, mais auraient suivi les traces d'un parent établi en Grèce avant la Seconde Guerre mondiale ; d'autres sont restés dans la région en s'appuyant sur les facilités que procuraient la présence dans la région de nombreux Grecs albanophones. Envisagés à cette échelle, le processus d'installation des immigrés fait intervenir des éléments nouveaux renvoyant à des solidarités inattendues entre les habitants des deux pays.

\section{Quelles solidarités possibles?}

Les relations entre la Grèce et l'Albanie étaient impensables durant la période du régime hoxhiste. Jusqu'en 1991, la frontière était hermétiquement fermée par une clôture électrifiée et toute personne voulant la franchir le faisait au péril de sa vie. Les liens pouvant être à l'origine de réseaux migratoires actuels procèderaient donc de la période précédant la Seconde Guerre mondiale. Les relations entre les espaces aujourd'hui situés dans les deux pays sont anciennes et on peut établir une série d'hypothèses concernant les solidarités possibles entre ceux d'“ici" et ceux de l'“autre coté". 


\section{Illustration 1}

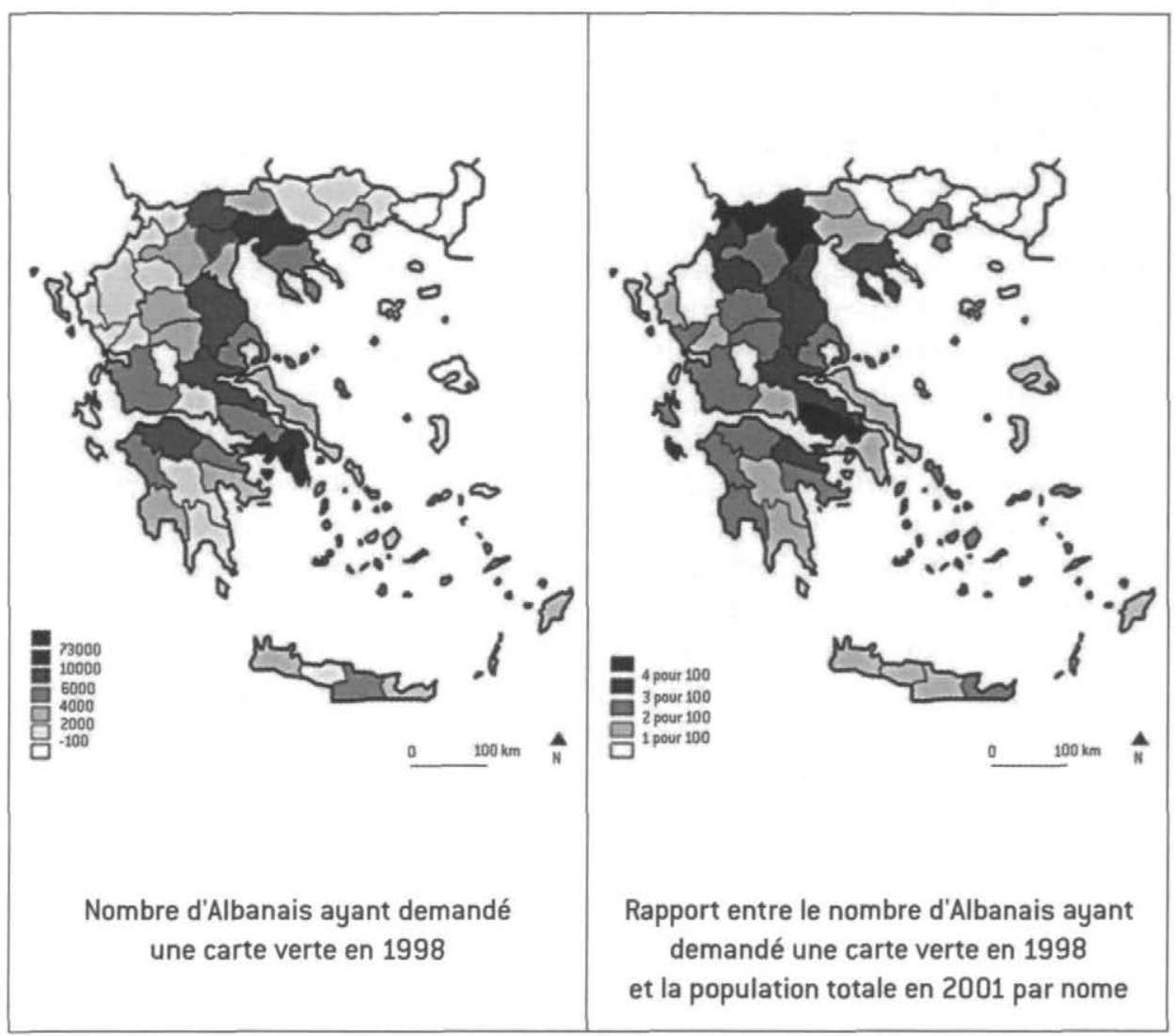

\section{Minoritaires, Valadues et Orthodoxes}

Du coté des Albanais, trois groupes paraissent liés à la Grèce par la langue et l'histoire. La minorité hellénophone d'Albanie méridionale est reconnue de manière très officielle par les gouvernements grec et albanais, mais sa taille demeure controversée. Selon les estimations et surtout la définition qui est retenue, elle peut varier de 60000 (gouvernement albanais en 1990) à 400000 pour les nationalistes grecs ${ }^{10}$. L'appartenance à ce groupe a facilité la migration. On reconnaît volontiers à Athènes qu'une centaine de milliers d'entre eux ont immigré en Grèce (Ministère de l'Intérieur) depuis 1991 ayant bénéficié de

10 Les chiffres maxima procèdent de l'hypothèse radicale que l'ensemble des chrétiens orthodoxes d'Albanie seraient des Grecs albanisés de force sous Enver Hoxha. Pour les débats concernant l'importance de la minorité grecque, voir Zarrineli (Luca), Albania, geografia della transizione, Milan : Francoangeli (collection Geografia e societa), 1999, p. 73. 
nombreux avantages pour venir s'établir dans la "mère patrie"11. Ces facilités sont surtout administratives : obtention systématique de visa ou d'une carte certifiant leur origine grecque. Les avantages que constitue la connaissance de la langue ne sont pas à négliger non plus.

Les Aroumains constituent le deuxième groupe du sud albanais ${ }^{12}$ à bénéficier d'avantages similaires. Ceux-ci sont descendants de pasteurs transhumants qui étaient présents dans toute l'Épire et en Macédoine. Leur particularité dans cette région est l'adoption de la langue grecque. En effet, si la langue domestique est demeurée l'aroumain, les membres de ce groupe savaient / savent le grec, la plupart de leurs terres de parcours se situant dans des régions hellénophones (Dropull, région de Sarandë). La présence de groupes aroumains en Grèce (Épire, Macédoine, Grèce centrale) donneraient aux Valaques d'Albanie une ressource supplémentaire pour faciliter leur installation en Grèce. Mais l'aspect déterminant est encore la situation administrative que leur accorde le gouvernement grec. Ils sont en effet considérés comme ayant une origine grecque (de sang grec-omogéneis) ce qui leur permet d'accéder aux mêmes facilités que les membres de la minorité grecque.

Le dernier groupe lié à la Grèce est plus large et englobe les deux précédents. Il s'agit de l'ensemble des orthodoxes d'Albanie qui peuvent bénéficier d'une solidarité religieuse supposable, mais aussi de l'idée fort répandue que tous les Albanais orthodoxes sont des Grecs albanisés de force par le régime d'Enver Hoxha. Cette vision des choses est inséparable de l'ancien contentieux territorial entre Grèce et Albanie autour de la question de l'“Épire du Nord"13. C'est grâce à cet héritage des conflits passés et à la faveur de mariages inter-communautaires (véritables ou inventés), que bon nombre d'orthodoxes d'Albanie ont aussi pu obtenir des documents d'omogéneis. Ce discours englobant l'ensemble des orthodoxes albanais à la nation grecque n'est pas si rare et il explique les chiffres avancés par les autorités grecques concernant les omogéneis venues d'Albanie.

\section{TChams et ARVANites}

Du coté grec, les groupes liés à l'Albanie sont nettement plus difficiles à cerner. Ceci tient à l'histoire nationale grecque et à ses conséquences sur les catégories administratives utilisées ${ }^{14}$. Les derniers chiffres sont ceux du recense-

\footnotetext{
${ }^{11}$ Ce chiffre est à considérer avec prudence car il prend en compte l'ensemble des personnes s'étant déclaré d'origine grecque et dépasse largement le cadre de la minorité officiellement reconnue.
}

${ }^{12}$ Pour la définition des groupes valaques ou aroumains, voir Gossiaux (Jean-François), Pouvoirs ethniques dans les Balkans, Paris : PUF, 2002, Pp. 131-148.

${ }^{13}$ L'Épire du Nord est le nom donné à la zone du sud de l'Albanie allant de la frontière grecque au fleuve Shkumbi qui fut revendiquée anciennement par la Grèce et encore actuellement par les nationalistes grecs.

14 Labbé (Morgane), “ Dénombrer les minorités : enjeux politiques et jeux des catégories dans les recensements grecs ", in Rallu (Jean-Louis), Courbage (Youssef), Piché (Victor), eds., Old and New Minorities, Paris : Eurotext / INED, 1997. 
ment de la population grecque de 1951 qui indiquaient pour le pays un total de 22736 albanophones soit $3 \%$ de la population du moment. Ces personnes étaient surtout concentrées dans les régions de l'Épire (les Tchams) mais aussi en Macédoine, en Grèce centrale et dans le Péloponnèse (les Arvanites).

La présence d'albanophones en Grèce remonte à la période précédant l'arrivée des Ottomans dans la région. Il s'agit de groupes installés à partir du XIII siècle que des bouleversements sociaux avaient contraint de fuir leur territoire d'origine ${ }^{15}$. Ces albanophones se sont maintenus jusqu'à nos jours même si l'on peut être sûr que leur particularité linguistique s'est atténuée au cours des années $^{16}$. Les Arvanites se définissent par des critères linguistiques et religieux : ils sont albanophones et orthodoxes. On les trouve essentiellement dans les zones de l'Argolide, de Béotie, en Attique, dans le Sud de l'Eubée et dans les îles du Golfe Saronique.

Les Tchams quant à eux, sont des albanophones présents uniquement en Épire, plus exactement dans la région comprise entre les fleuves Acheron et Vouthroto correspondant à l'actuelle Thesprotie (en Grèce) et la région de Konispol (en Albanie). En Grèce, on a coutume de dire que les Tchams se distinguent par la pratique de la religion musulmane. Pourtant, des récits de voyage révèlent que ce n'est pas toujours vrai. Athanasos Psalidas, au début du XVIII siècle décrit la région et la dit peuplée de Grecs et d'Albanais : "les premiers plus nombreux et les seconds se divisant entre Chrétiens et Musulmans"17. Il s'agit donc bien plus d'une communauté linguistique que religieuse. Après la Seconde Guerre mondiale, accusés de collaboration avec les armées d'occupation italienne et allemande, ainsi que de soutien dans l'accomplissement de massacres, la plupart des Tchams de la région se sont enfuis ou ont été expulsés ${ }^{18}$. Pourtant, certains albanophones ont pu demeurer dans le pays, mais essentiellement ceux qui étaient de confession orthodoxe. Compte tenu de la propension à assimiler l'identité grecque à l'orthodoxie dans cette région périphérique, il n'est pas étonnant que ces personnes aient été plus volontiers considérées comme des Grecs que comme les membres d'un groupe "minoritaire" traitre à la cause nationale ${ }^{19}$.

15 Ducellier (Alain), Doumerc (Bernard), Imhaus (Brunehilde), De Miceli (Jean), Les chemins de l'exil, bouleversements de l'Est européen et migration vers l'Ouest à la fin du Moyen-âge, Paris : Armand Colin, 1992.

${ }^{16}$ Guefou-Madianou (Dimitra), " Cultural polyphony and identity formation : negotiation tradition in Attica ", American Ethnologist 26 (2), 1999.

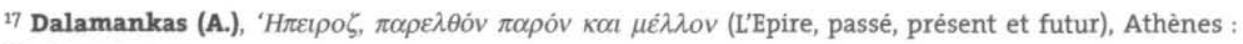
Koultoura, 2002.

18 Péchoux (Pierre-Yves), Sivignon (Michel), "L'éviction des Tchamidès d'Epire occidentale en 1944 ", Ethnographie (L'), 85-2 (106), 1989 ; Popović (Alexandre), L'Islam balkanique. Les musulmans du sud-est européen dans la période post-ottomane, Berlin / Wiesbaden : Otto Harrassowitz, 1986 ; Prévélakis (Georges), Géopolitique de la Grèce, Paris : Complexe, 1997, p. 39.

${ }^{19}$ Cf. Mihalopoulou (D.), Tб夭́ $\mu \eta \delta \varepsilon \zeta \zeta$ (les Tchams), Athènes : Arsenidis, 1993. 
Le fait est que sur la vingtaine de milliers d'albanophones présents dans l'Épire grecque avant guerre, il n'en restait que 11 ooo en 1951 et comme le montre le document $\mathrm{n}^{\circ} 2$, ce sont essentiellement les musulmans qui ont quitté du pays.

\section{Document 2 : Citoyens grecs albanophones recensés en 1940 et 1951}

\begin{tabular}{|l|c|c|c|c|c|}
\hline & Orthodoxes & Proportion & Musulmans & Proportion & TOTAL \\
\hline 1940 & 32712 & $65 \%$ & 16890 & $25 \%$ & 49632 \\
\hline 1951 & 22207 & $98 \%$ & 487 & $2 \%$ & 22736 \\
\hline
\end{tabular}

Source : $E S Y E$

Il apparaît donc qu'aussi bien du coté albanais que du coté grec, il est possible de trouver des groupes de personnes liés de différentes manières aux habitants du pays voisin. Ces liens sont-ils pour autant à l'origine de solidarités pouvant faciliter ou orienter les flux migratoires actuels ? Difficile de répondre tant les groupes décrits ci-dessus se situent à des degrés de proximité différents. Ainsi, il apparaît discutable de comparer les Arvanites, présents en Grèce depuis une époque antérieure à l'émergence du sentiment national albanais, et les Vorio-épirotes ${ }^{20}$, qui peuvent encore avoir de la famille proche en Grèce. Dans chacun des cas, la nature du lien avec l'autre (l'Albanais ou le Grec) sera extrêmement différente. À l'opposé des solidarités, ce sont même des formes de rejets qui peuvent s'opérer, comme dans le village arvanite de Koropi en Attique où les locaux ont commencé à tenir un discours bien plus radical sur leurs racines grecques depuis l'arrivée des immigrés albanais, dont ils veulent se différencier à tout prix. Pourtant, la réalité peut être bien plus complexe et il n'a pas été rare de constater des formes de rapports privilégiés entre Arvanites et Albanais. Mais dans le cas des réseaux frontaliers que je vais examiner ci-dessous, les choses sont encore plus immédiatement saisissables puisque ce sont des formes de réseaux familiaux, courants dans le cas de la migration albanaise, qui se colorent ici d'une teinte très particulière.

À la grande variabilité des cas de figure s'ajoute la question de la représentativité et du poids de ces hypothétiques réseaux sur le cours de la migration. S'il convient de ne pas sous estimer l'impact de ces liens sur l'ensemble de la migration - les relations n'étant pas minces entre les Albanais arrivés en Grèce grâce à leur connaissance du pays et ceux qui sont venus sans avoir de contact préalable, comme le montre le roman de V. Graçi ${ }^{21}-$, reconnaissons néanmoins que considérer les mouvements migratoires consécutifs à l'exis-

\footnotetext{
${ }^{20}$ Cette expression signifie "Épirote du Nord". C'est ainsi que l'on désigne les hellénophones d'Albanie en Grèce alors que les Albanais les appellent "minoritaires".
}

${ }^{21}$ Graçi (Virion), Au paradis des fous, Tour d’Aigues : Éditions de l'Aube, 1998. 
tence de ces solidarités comme des cas particuliers ramène leur étude à l'examen d'un point de détail négligeable, une goutte d'eau dans la marée migratoire qui a conduit 500000 Albanais en Grèce. Dans ce cas, pourquoi les étudier ? Cette question rejoint plus largement la manière d'envisager l'étude des migrations. Considérer "globalement" les migrations, selon les propres dires de G. Simon, est une nécessité22. Pour ce faire, on en vient à réhabiliter le "migrant" et son parcours comme élément constitutif de la complexité du phénomène ${ }^{23}$. Dans cette perspective, le cas particulier de la frontière examiné ciaprès permettra d'illustrer la manière dont l'identité est utilisée comme ressource dans la migration et comment cet élément stratégique vient jouer dans les logiques de répartition et de mobilités des Albanais en Grèce. Même s'ils ne concernent qu'un nombre limité de personnes, ces réseaux permettent de mettre en avant, dans les situations les plus visibles, des processus communs à l'ensemble du flux. Ce parti pris méthodologique, qui consiste à éclairer le général par le particulier, vise à faire une place centrale aux acteurs dans le système de choix qui constitue leur histoire migratoire. C'est donc pour ces deux raisons, méthodologique et informative, que se justifie l'étude détaillée des réseaux transfrontaliers proposée ci-dessous.

\section{DES RÉSEAUX TRANSFRONTALIERS : LUNXHËRI, POGON, DROPULL}

Pour certains groupes vivant dans la zone frontalière autour de la ville albanaise de Gjirokastër, la Grèce en 1991 n'était pas tout à fait une terra incognita. En effet, de nombreux habitants de cette région sont apparus liés à ce pays par leur histoire familiale. Les pages qui suivent sont le résultat d'enquêtes répétées auprès de plusieurs communautés "transfrontalières" depuis plus de deux ans : les membres de la communauté hellénophone du Pogon et du Dropull, d'une part, et, de l'autre, les Valaques de la région de Gjirokastër, rencontrés plus particulièrement en Lunxhëri, dans le Pogon et à Gjirokastër même.

\footnotetext{
${ }^{22}$ Simon (Gildas), " Penser globalement les migrations ", Projet, (272), hiver 2002-2003.

${ }_{23}$ C'est le "changement de paradigme" énoncé par $M$. Gauchet et appliqué à la recherche géographique - dans ce cas, à l'étude des mobilités géographiques (Lévy (Jacques), Le tournant géographique, Paris : Belin (collection Mappemonde), 1999, p. 112) dont un exemple magistral est donné par les travaux concernant la migration portugaise écrits par Y. Charbit, M.-A. Hily et M. Poinard (Charbit (Yves), Hily (MarieAntoinette), Poinard (Michel), Le va et vient identitaire, migrants portugais et village d'origine, Paris : INED / PUF, 1997.
} 


\section{Des liens historiques et humains pour une région transfrontalière}

Toute la zone, avant la fermeture de l'Albanie en 1945, était le lieu d'échanges intenses. Non seulement, le franchissement de la frontière ne posait pas de problème particulier, mais l'imbrication des modes de vie, des langues (grec, albanais ou aroumain), des propriétés et des familles en faisait un espace qui, sous certains aspects, connaissait un fonctionnement intégré du point de vue économique et humain ${ }^{24}$. Espace de passage entre les deux pays, l'ensemble de la région était commandé par la ville de Ioannina et, à un degré moindre, par Gjirokastër.

\section{ILLUSTRATION 2}

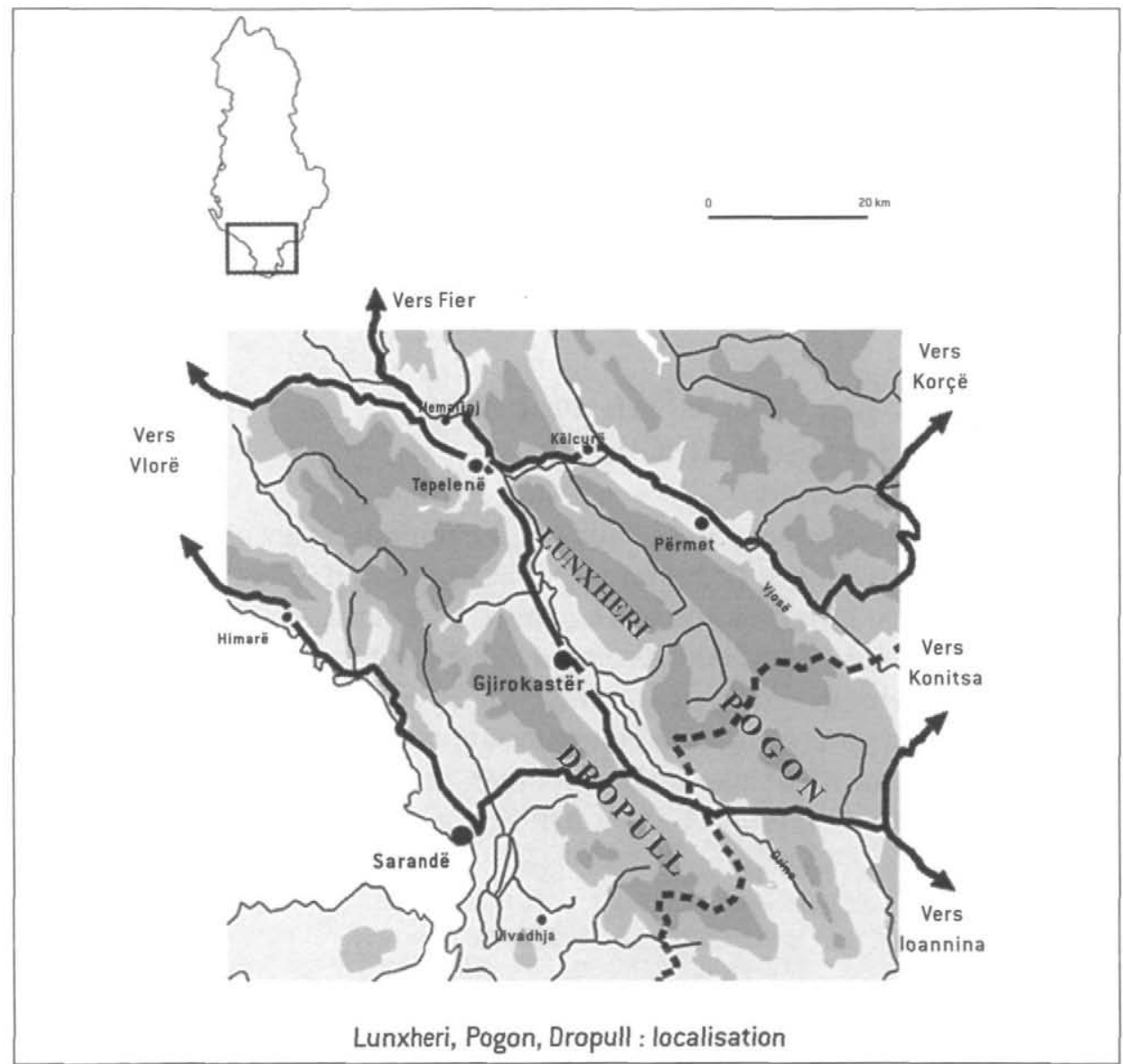

24 On parlera ici de fonctionnement intégré pour les groupes transfrontaliers plutôt que d'espace intégré pour la zone concernée car d'autres personnes vivant dans ces régions connaissaient de sévères contrôles pour franchir la frontière. Ici comme ailleurs, l'espace est affecté de textures différentes selon l'appartenance à tel ou tel groupe, confirmant le lien indissoluble entre intégration sociale et intégration spatiale (Rhein (Catherine), " Intégration sociale, intégration spatiale ", Espace géographique (L'), (3), 2002). 


\section{DES LIENS INDISCUTABLES}

Que les habitants fussent de purs sédentaires ou des transhumants, la frontière n'avait ici que peu de poids dans l'organisation de leur espace de vie au regard des caractéristiques linguistiques ou religieuses des populations.

Les groupes les plus mobiles de la région étaient les populations valaques. Il s'agissait de pasteurs semi-nomades qui, selon la logique de la transhumance inverse, passaient l'hiver dans des cabanes dans la région de Ksamili, face à Corfou, entre Sarandë et le site archéologique de Butrint, ou en Thesprotie, entre Parga et Igoumenitsa, et se rendaient une partie de l'été dans le massif de Kolonjë entre Përmet et Korçë. Ce déplacement n'a rien d'étonnant pour les Valaques et reste conforme à ce que décrit J. Cvijić :

"Ces migrations sont surtout pratiquées par des pasteurs aromounes, moins par les Albanais, moins encore par les Slaves... Tous ces pasteurs s'acheminent avec leurs troupeaux, à la fin du mois d'octobre, vers les régions où les pâturages ne font pas défaut en hiver"25.

Comme l'écrit J-F. Gossiaux, ces groupes avaient, au cours de leurs déplacements un point fixe ${ }^{26}$. Pour les Valaques de la région de Gjirokastër, il s'agissait du village de Képhalovrisso (anciennement appelé Medjidié). C'est en 1853 que les autorités ottomanes présidèrent à la création de ce village, appelé ainsi en l'honneur du sultan Abdulmédjid qui régna entre 1831 et $1861^{27}$, afin de contrôler une communauté largement gagnée à la cause grecque et hellénisée quelques années seulement après l'accession à l'indépendance de la Grèce moderne.

Dans la partie méridionale de la région, se fait sentir l'influence de l'église et du nationalisme grecs, soutenu en partie par les Aromounes hellénisés. Ces derniers devinrent les commerçants les plus actifs de toute la région, dans les villes et dans les nombreux marchés provisoires qui se tiennent autour des gués et aux embouchures des rivières une fois par semaine ou seulement aux jours de fête ${ }^{28}$.

Ce village, aujourd'hui en Grèce, était devenu le point d'attache de ces pasteurs valaques ${ }^{29}$. Il se situe à mi-distance entre les deux points de leur parcours annuel, mais dans une zone montagneuse qui en fait un lieu d'estive au moins

\footnotetext{
${ }^{25}$ Cvijić (Jovan), La pénínsule balkanique, géographie humaine, Paris : Armand Colin, 1918, p. 178.

26 "L'habitat fixe était celui de l'été, en un endroit précis de la montagne habité d'année en année ". Gossiaux (Jean-François), op.cit, p. 141.

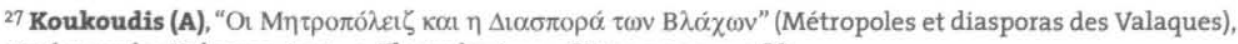
Etudes sur les Valaques, tome 2, Thessalonique : Zitros, 2000, p. 288.
}

${ }^{28}$ Cvijić (Jovan), op.cit., p. 79

29 Képhalovrisso demeure le point d'attraction comme de ralliement "identitaire" et d'auto-désignation : encore actuellement, l'association des Valaques de la région de Gjirokastër s'appelle "Képhalovrisso". 
intermédiaire. La plupart d'entre eux y naissaient et y construisaient des maisons qu'ils n'occupaient qu'une partie de l'année. Au moment de l'établissements des États grec et albanais en Épire, les comportements de ces pasteurs ne se modifièrent pas et ils continuèrent à parcourir le pays (les États), de la côte à la montagne, avec une préférence pour les terres situées dans des zones hellénophones (Dropull, Pogon, région de Sarandë) mais pouvant, quand les prix de location ou la qualité des terres l'exigeaient, passer par des zones albanophones comme la Lunxhëri ou la Thesprotie. Les Valaques de Képhalovrisso connaissaient des sous-groupes de taille importante, les soj, familles élargies, regroupées pour les déplacements en tsélinga comprenant 30 à 100 personnes (armées), portant le nom du tsélingatos qui possédait le plus de têtes de bétail.

La population hellénophone de la région est - quoique sédentaire -, elle aussi, une population de la frontière. En effet, contrairement aux régions de Sarandë ou de Himarë, les groupes du Pogon et du Dropull sont directement adjacents au territoire de l'État grec. Ils forment ainsi l'extrémité d'un continuum linguistique tranché aujourd'hui par la frontière ${ }^{30}$. On ne retrouve ce cas que dans les quelques villages hellénophones situés à flanc de montagnes en rive droite de l'Avios, au sud de Përmet. Dans le Pogon comme dans le Dropull donc, la frontière de 1913 est venue diviser un ensemble humain. Ainsi, le nom même de Pogon est-il utilisé pour désigner l'ensemble de la région située de part et d'autre de celle-ci regroupant 38 villages en Grèce et 5 en Albanie qui formaient un seul et même kaza sous l'administration ottomane avec pour capitale le village de Vochtine (actuel Pogoniani). De la même manière, le costume traditionnel du village de Sopik (coté albanais) est semblable à celui des villages grecs de Pogoniani, Delvinaki et Vissani ${ }^{31}$. Surtout, des mariages se nouaient entre personnes des différents villages, même après l'établissement de la frontière en 1913. Ceci aura une importance considérable par la suite.

Avant l'établissement du régime Enver Hoxha en Albanie, le franchissement aisé de la frontière permettait à des personnes de posséder des terres de l'autre côté et d'aller les cultiver chaque jour. De même, les gens du Pogon grec avaient coutume de se rendre à Gjirokastër pour le marché hebdomadaire, ou prenaient le bateau à Sarandë pour rejoindre Athènes, Istanbul ou l'Amérique. Il faut dire qu'au temps de l'Empire ottoman, les hellénophones de la région étaient partis en nombre vers Istanbul ${ }^{32}$. Les migrants de chaque village se

\footnotetext{
$3^{\circ}$ Dans la partie orientale de la frontière albano-grecque, la situation a été profondément bouleversée par les échanges de population - faisant de la limite politique une frontière ethnolinguistique (De Rapper (Gilles), Deslondes (Olivier), Roux (Michel), " Dimanche à Miras, lundi à Dipotamia, la frontière albanogrecque dans la région de Bilisht et de Kastoria ", Cahiers d'études sur la Méditerranée orientale et le monde turco-iranien, (29), janvier-juin 2000).
}

${ }^{31}$ Les costumes de ces villages sont blancs alors que l'on porte le noir dans les régions environnantes.

${ }^{32} \mathrm{Cf}$. De Rapper (Gilles), Les Albanais à Istanbul, Istanbul : Les dossiers de l'IFEA, 2000. 
spécialisaient alors dans un corps de métier ${ }^{33}$. Ainsi, les gens de Vissani étaient-ils majoritairement des boulangers. Les émigrés partaient seuls, laissant femme et enfants au village. Ils conservaient cependant des liens étroits avec leur petite patrie par l'intermédiaire de fraternités et participaient financièrement à des travaux dans le village pour leur propre maison, comme le constate Parmentier dans ces villages d'Albanie du Sud, ou à l'usage de la communauté (moulin, pont, église, école...). "On rencontre parfois des somptueuses demeures qui remplacent les anciennes forteresses, et qu'habitent des Albanais enrichis qui sont revenus, dans la patrie, après fortune faite" 34 .

Ce groupe a aussi été l'acteur d'une migration transocéanique vers les États-Unis, l'Argentine et l'Australie à partir du début du vingtième siècle au moment où la situation économique de l'Empire ottoman commença à décliner et après sa disparition. Dans cette migration, les hellénophones originaires des villages situés du côté albanais de la frontière ne se distinguaient pas des autres Grecs et il n'était pas rare que fortune faite, ils investissent indifféremment de part et d'autre de la frontière ou rentrent "au pays" en s'établissant à Athènes ou à Corfou.

\section{LE "TEMPS des balles"}

Après les épisodes de la Seconde Guerre mondiale (offensive italienne, contre-offensive de l'armée grecque et repli de l'armée allemande), la zone a connu une nouvelle période troublée jusqu'à la fin de la guerre civile grecque. Les membres de la communauté grecque d'Albanie, gagnés aux communistes se battirent souvent auprès des partisans de l'EAM. Mais, une fois la guerre terminée et la victoire des forces gouvernementales acquise, l'Albanie et la Grèce adoptèrent l'attitude de pays en état de guerre (maintenu jusqu'en 1987 et partiellement levé aujourd'hui) et l'espace frontalier s'est défini en conséquence.

Néanmoins, la frontière ne se ferma pas partout immédiatement, ce qui permit à de nombreuses personnes de fuir un régime qu'ils pressentaient dictatorial. Ainsi, les fuites se sont multipliées jusqu'au début des années 1950. On raconte souvent dans le Pogon l'histoire de ces émigrés partis en Amérique qui payaient alors des hommes de main installés du coté grec pour qu'ils aillent dans les villages du Pogon albanais chercher leur famille afin qu'elle puisse partir les rejoindre outre Atlantique. De la même manière, en raison de la collectivisation 35 , de nombreux pasteurs valaques profitèrent de leur connaissance de la montagne pour s'enfuir en Grèce et éviter ainsi que leurs bêtes ne soient saisies.

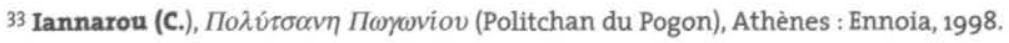

34 Parmentier (E.), Voyage dans la Turquie d'Europe, Paris : Ernest Leroux, 1890 [2e édition], p. 266.

35 La collectivisation fut entamée en 1948 dans le Pogon. 
Une telle situation poussa le gouvernement d'Enver Hoxha à adopter des mesures strictes de contrôle de la frontière. À partir de 1955, toute la famille des fuyards était envoyée en exil afin de dissuader les autres villageois. Une zone de terre meuble sans cesse retournée servit tout d'abord à détecter les mouvements dans la zone, puis une clôture électrifiée (le klon) fut construite au-delà de laquelle, toute personne non autorisée pouvait-être abattue sans sommation $^{36}$. Un tel dispositif permit de juguler presque totalement tout passage de la frontière par la montagne et il resta en vigueur jusqu'en 1990 et la chute du régime hérité de l'Oncle Enver. Cette situation suspendit ainsi toute communication entre les deux côtés de la frontière.

Mais les autres types de communication étaient eux-aussi interdits et toute personne suspectée d'avoir des contacts avec la Grèce pouvait être inquiétée par les autorités. Les Valaques firent les frais de cette politique dans des proportions considérables. En effet, ceux qui se trouvaient du côté albanais au moment de la fermeture de la frontière furent contraints d'y rester. Quand la politique de collectivisation fut appliquée, ils furent assignés à une coopérative et sédentarisés dans les villages de la zone. C'est ainsi que des Valaques de "Képhalovrisso" se retrouvent dans quasiment tous les villages de la région de Gjirokastër 37 .

Leurs liens avec la Grèce étaient si importants que dans le Pogon, les Valaques s'étaient déclarés membres de la minorité grecque et recevaient l'enseignement dans cette langue. Ce n'est qu'à partir de 1966 qu'ils ont été poussés à changer de groupe national pour devenir des "Albanais". Malgré cette "conversion", les Valaques étaient toujours perçus comme les "amis des Grecs" et, à cet égard, suspects de sympathie pour l'impérialiste voisin. Persécutions et humiliations ont été des éléments de leur quotidien durant ces années et ils furent nombreux à connaître la déportation. Suspicion et racisme se mêlaient alors pour faire des Valaques - pourtant très nombreux - voire majoritaires dans la plupart des villages de Lunxhëri - les personnes les plus déclassées et les plus touchées par la violence de la "lutte des classes" initiée par Enver Hoxha dans les années 1950.

Sur la place de Mingull (Lunxhëri), Nikola ne tarit pas en cet après-midi pour nous raconter les humiliations que les Valaques du village ont subies sous le régime précédent.

Sous Enver Hoxha, tous les membres de mon lignage n'avaient pas le droit d'aller à l'école après les 8 ans d'instruction élémentaire. A la ferme, on leur attribuait les

${ }^{36}$ Ces mesures de renforcement de la frontière n'ont pas été appliquées simultanément puisque ce n'est qu'en 1975 que le klon est érigé dans le Pogon alors qu'il apparait dès le début des années 1960 dans le Dropull et la vallée de l'Avios.

37 On les retrouve essentiellement dans les villages d'Anton Poçi, de Suha et globalement dans toute la Lunxhëri en particulier dans les villages de Nokov et de Mingull. Ils sont absents des villages du Dropull et de Sopik dans le Pogon. 
travaux les plus durs et ils n'avaient pas le droit de se marier avec des gens à bonnes biographies $^{38}$.

Quand une voiture arrivait au village, on avait peur et on s'enfermait à double tour. Une fois qu'elle partait, on passait de maison en maison pour savoir qui elle était venue chercher. Ma mère, une fois est allée au magasin chercher du sucre et le responsable l'a renvoyée en lui disant : "du sucre, tu n'as qu'à aller en chercher chez ton père, en Grèce !". Nous n'avions pas de frigo ni de machine à laver alors que les communistes en avaient. On dit que c'était la dictature du prolétariat mais en fait, c'était la dictature de ceux qui avaient une bonne biographie et qui servaient le régime sur les autres.

Durant cette période, les membres de la communauté grecque étaient euxaussi inquiétés mais dans des proportions moindres, dans la mesure où le gouvernement hoxhiste voulait se servir de ce groupe comme d'un argument de négociation avec le pays voisin. Pourtant, ceux dont un membre de la famille s'était enfui en Grèce étaient passibles d'enfermement de la même manière.

\section{UNE ÉVOLUTION DISJOINTE}

Une fois la fermeture de la frontière instaurée, les deux parties se sont trouvées incluses dans des logiques très différentes. Ce fait provoqua une évolution radicalement opposée pour ces espaces au fonctionnement pourtant comparable au départ.

Du côté albanais, les mouvements de population, s'il on excepte les rares fuyards qui réussirent à franchir le klon, ne se font qu'au sein de l'espace national. Ces mouvements sont, par ailleurs, restreints du fait de la loi. Ainsi, l'exode rural observé pour le pays dans les années qui suivirent l'après-guerre est stoppé par un arsenal législatif coercitif (à partir de 1961) et les ruraux, dans les faits, se sont trouvés attachés à leur village afin de "faire les collines et les montagnes aussi fertiles que les plaines". Les seules personnes autorisées à se déplacer sont les femmes qui partaient se marier dans un autre village (mais généralement, ces mobilités sont de faible distance), les étudiants et les ingénieurs mutés vers d'autres villes.

Du fait de la suspicion qui pesait sur les membres de la minorité grecque et sur les Valaques de la région, ces groupes étaient soumis à un autre type de mobilité - forcée celle-là - par les nombreux internements et exils qui leur ont été imposés 39 . Globalement, toute la région a connu, à cette époque, une aug- 
mentation substantielle de sa population du fait d'un accroissement naturel important et d'un bilan migratoire presque nul.

Ce n'est pas le cas des régions voisines situées en Grèce : leurs habitants ont suivi les logiques "internes" de mobilité de la population grecque et la région s'est largement vidée à l'instar du nome de Ioannina et de l'ensemble de l'Épire (voir document $\left.n^{\circ} 3\right)^{40}$. Les villageois sont partis essentiellement pour Athènes et l'étranger ${ }^{41}$. Aujourd'hui, la zone couverte par l'éparchie du Pogon demeure une des régions d'Europe qui connaît les indicateurs de développement les moins favorables. Même si l'on constate en Epire un certain nombre d'installations en relation avec le dynamisme dans ses centres urbains, la population des zones rurales de la frontière continue de fléchir. En tout et pour tout, les villages du Pogon (grec) ont vu leur population diminuer de plus de $30 \%$ et se concentrer dans les principaux villages. L'attraction vers ces villages s'est vue conforter par la réforme "Kapodistria" de 1991, élevant certains d'entre eux au rang de chef-lieu de municipalité (Delvinaki et Képhalovrisso).

\section{Document 3 : Evolution de la population épirote}

\begin{tabular}{|l|r|r|r|r|r|r|}
\hline & \multicolumn{1}{|c|}{1951} & \multicolumn{1}{|c|}{1961} & \multicolumn{1}{c|}{1971} & \multicolumn{1}{c|}{1981} & \multicolumn{1}{c|}{1991} & \multicolumn{1}{c|}{2001} \\
\hline Grèce & 7632801 & 8388553 & 8768641 & 9740417 & 10259900 & 10964810 \\
Epire & 330543 & 352700 & 310334 & 324541 & 339728 & 353820 \\
Ioannina & 153748 & 155326 & 134688 & 147304 & 158193 & 177137 \\
\hline Pogon & 9453 & 8510 & 5886 & 6887 & 6442 & 6315 \\
\hline
\end{tabular}

Source : $E S Y E$

Comparer la situation dans les parties albanaise et grecque du Pogon en 1990 montre à quel point les cinquante années de fonctionnement dissocié ont établi un contraste plus que prononcé. Du coté grec, les villages se sont vidés au point que la densité de l'éparchie du Pogon est passée de 20 à 13 habitants au kilomètre carré entre 1951 et 1991, alors que le côté albanais la voyait augmenter jusqu'à plus de 40 . Il en va de même dans le Dropull : au moment où les villages frontaliers du coté grec se dépeuplaient, un nouveau centre de service était crée ex-nihilo avec le village de Vrissera qui devenait le centre d'une coopérative de grande taille.

Ainsi, après avoir connu une certaine communauté de destin, les habi-

40 Pteroudis (Evangelos), " Émigrations et immigrations en Grèce : évolution récente et questions politiques ", Revue Européenne des Migrations Internationales, 12 (1), 1996.

${ }^{41}$ Pour la région de Ioannina, plus de $70 \%$ des mobilités demeurant dans le pays se faisaient au détriment des zones rurales dont $22 \%$ vers Athènes et $15 \%$ vers Ioannina, en 1971 . Les mouvements internationaux ont aussi durement frappé les régions de la frontière : dans les années 1960,60\% de la population de Képhalovrisso serait partie en Allemagne fédérale vers les villes de Zingelfingen, Menden et Wuppertal. 
tants de la zone, Grecs du Pogon et du Dropull ou Valaques originaires de Képhalovrisso, ont été séparés par la frontière et ont été contraints d'adopter des "modes de vie" différents : pour les uns, celui du néo-citadin ou du Gastarbeiter, pour les autres, celui du paysan socialiste. Mais, à partir de la chute du régime, les habitants des deux côtés se sont retrouvés et leurs anciens liens ont eu l'occasion de rejouer.

\section{Le choc migratoire des années 1990}

La fin de l'économie planifiée et du régime qui l'imposait en Albanie a eu pour conséquence l'ouverture de la frontière avec la Grèce. Très rapidement, les Albanais ont fait disparaître tout ce qui pouvaient rappeler le régime. Les statues aussi bien que les bâtiments collectifs ont été dévastés et, en quelques jours, la clôture qui séparait les deux pays fut détruite en mai 1991. La conséquence immédiate fut le départ vers la Grèce d'un nombre considérable d'Albanais ${ }^{42}$. Parmi ceux-ci, les habitants de l'Albanie du sud ne furent pas en reste et bon nombre d'entre eux passèrent de l'autre côté, mais, dans leur cas, le voyage ne se faisait pas toujours à l'aveuglette.

\section{REPRISE DE CONTACT}

Néanmoins, la reprise des liens de part et d'autre de la frontière avait débuté depuis quelques années. A partir de 1987, avec la levée partielle de l'état de guerre, la frontière fut entrouverte et certaines personnes avaient pu venir de Grèce pour rendre visite à leurs parents vivant du coté albanais. Mais au moment de l'ouverture de 1991, les récits que l'on a pu recueillir décrivent un élan mutuel qui amenait les retrouvailles enthousiastes de familles séparées depuis cinquante ans.

1- Sur la place de Képhalovrisso, Kostas nous raconte, devant un tsipouro et malgré le soleil de cet après-midi du mois d'août, quelle fut la réaction des gens de sa famille quand ils ont appris l'ouverture prochaine de l'Albanie.

Quand nous avons appris que la frontière allait être ouverte, nous sommes partis immédiatement vers Kakavia (le poste frontière sur la route entre Ioannina et Gjirokastër). On y a rencontré ma tante paternelle que je n'avais jamais vue jusqu'alors. Puis les cousins sont arrivés par la montagne. C'était une vrai route. Il y avait des milliers d'Albanais qui passaient. Les cousins sont restés là plus d'une année. On a appris à se connaître et ils sont partis ailleurs.

2- Mais ces retrouvailles n'ont pas eu lieu uniquement sur les points de passages officiels. Ainsi, dans la vallée de l'Avios, c'est sur un sentier qui serpente à flanc de montagne que les familles se sont retrouvées entre les villages de Psiloterra et Molivdokepastos.

${ }^{42}$ Sintès (Pierre), " La migration des Albanais en Grèce, difficultés méthodologiques pour une étude géographique ", Revue Européenne des Migrations Internationales, 17 (3), 2001. 
Quelle ne fut pas la surprise de Dimitri quand il entendit ce matin là la clarinette sur ce sentier qui lui avait été interdit toute son enfance à cause des gardes frontières albanais. Il ne le croyait pas mais c'était bien çà, les gens de Psiloterra, le village hellénophone situé de l'autre coté de la frontière étaient en train de monter sur la route du monastère. Quand ils sont arrivés à leur hauteur, c'était vraiment comme une fête pour tous. L'un d'entre eux se présenta à lui avec trois noms griffonnés sur un bout de papier. L'un de ces noms ètait celui de sa mère. Lui, était un cousin. Il ne l'avait jamais vu.

Une fois ces retrouvailles faites, les départs se sont multipliés depuis l'Albanie. Tous les candidats au départ comptaient s'appuyer sur les contacts qu'ils avaient en Grèce pour faciliter leur migration. Pour les Valaques et les hellénophones du Pogon, cela signifiait se rendre dans les villages du Pogon grec pour rechercher leurs contacts. C'est pourquoi tous passaient par le chemin qui mène de Sopik à Drimades, à travers la montagne. Inutile de dire que ce passage n'était pas, jusqu'à une date très récente (juillet 2002), contrôlé par les autorités des deux pays et les franchissements se faisaient dans l'irrégularité43. Les Valaques se rendaient à Képhalovrisso pour y retrouver leur famille et les autres allaient vers les villages de Pogoniani et de Delvinaki où avaient été aménagés des centres d'accueil.

Les départs se faisaient toutes générations confondues contrairement aux autres migrants qui quittaient alors l'Albanie. Ces derniers étaient en majorité de jeunes hommes capables de supporter les difficultés d'un passage clandestin de la frontière, mais aussi un premier séjour souvent inconfortable en Grèce. Ce n'est qu'une fois leur situation assurée que des personnes plus âgées ou leur famille les ont suivis. Dans le cas des frontaliers, l'importance des relations familiales donna un rôle bien différent aux personnes âgées qui partirent parfois en premier pour renouer les liens avec leurs parents.

L'accueil dans les villages grecs fut variable. Les cas de figure sont multiples d'une nuit passée chez de lointains parents dans la gêne avant de repartir le lendemain matin à une prise en main totale par un lointain grand-oncle qui poussa la fidélité familiale jusqu'à perdre la vie sur les périlleuses routes albanaises pour aller chercher des visas à l'ambassade de Grèce à Tirana. Mais, en règle générale, quel que fut l'accueil des parents "grecs", leur village a servi immanquablement de premier pas (proto vima) ou de première base (proti vasi). On s'y est souvent établi quelques temps (entre un mois et un an) avant de trouver d'autres destinations. Il faut bien dire que le travail manque toujours dans la région. Ce n'est pas pour rien qu'elle a été désertée par ces habitants dans les décennies précédentes et peu nombreux sont les "Épirotes du Nord" à 
s'être installés dans les villages de la frontière (on n'en trouve qu'une vingtaine de famille à Képhalovrisso, 35 personnes à Delvinaki, 6 à Pogoniani et 8 à Vissani - en général, moins que des "Albanais" non omogéneis). En revanche, les groupes se déplaçant à la suite des pionniers peuvent être d'une taille considérable.

Quand Ari est parti la première fois, il est passé par Sopik et Képhalovrisso. Il ne s'est pas attardé car il savait où aller : il a pris un taxi pour Trikala où l'attendait un oncle. Il vit maintenant à Trikala avec une cinquantaine de personnes de sa famille.

\section{SUIVRE UNE MIGRATION FOSSILE}

À partir de là, des immigrants se sont orientés vers des destinations où ils étaient susceptibles de retrouver des connaissances ou de la famille, ils ont donc suivi à la traces des personnes parties de Képhalovrisso ou des autres villages du Pogon durant les décennies précédentes. Les nouveaux migrants ont reproduit alors le comportement des villageois épirotes des années 19501970 en se dirigeant majoritairement vers Athènes et Ioannina et, parfois, vers des destinations marginales qui avaient été choisies, il y a vingt ou trente ans, par un parent inconnu (comme Larissa, Sparte ou Volos pour les Valaques de Képhalovrisso). Dans ce cas, l'appartenance à ces réseaux est un facteur déterminant dans le choix de la destination et doit être, pour ces groupes, prise en compte quand on cherche à en expliquer la répartition sur le territoire grec.

Pourtant on ne peut pas parler d'un schéma suivi automatiquement dans la mesure où son application dépend des comportements et des choix de chacun. Néanmoins, quelle que soit l'impression que laisse cette expérience aux migrants (amertume ou enthousiasme), l'aide apportée par le passage dans les villages de la frontière est finalement substantielle, ne serait-ce que dans le domaine administratif. En effet, la grande majorité en a profité pour se faire établir des certificats attestant de leur ascendance "grecque" qui leur a permis d'échapper à bien des tracasseries administratives lors des vagues de régularisation amorcées par le gouvernement grec à partir de 1997. Ils sont aujourd'hui titulaires d'une carte d'omogeneis et bénéficient, à ce titre, des avantages afférents.

1- La première fois que Giorgos est parti en Grèce, c'était pour Képhalovrisso. Il est allé voir un cousin mais celui-ci n'a pas pu l'aider. Il n'y est plus jamais retourné. Ça ne lui posait pas de problème d'avoir un visa à l'époque en tant que Valaque. Il a une carte d'omogéneis depuis 1999.

2- La première fois que Spiros est passé en Grèce, c'était par Sopik en 1991 avec un groupe de 15 personnes, tous Valaques. Sur la route, les soldats albanais les ont soulagés de tout ce qu'ils emportaient avec eux. Les soldats grecs les ont laissé passer car leur "petit passeport" portait la mention "nationalité grecque". Arrivés à Képhalovrisso, ils sont allés voir leur famille et on leur a donné à manger et de 
quoi s'habiller. Ils sont restés là une semaine et ils sont repartis vers Loutraki car certains parents qui vivent à Ioannina leur avaient conseillé l'endroit.

Ce type d'avantages a permis l'établissement de nombreuses personnes en Grèce. L'existence de ces réseaux est un facteur indéniable facilitant la migration. On constate, sans surprise, que ces groupes ont migré en masse vers la Grèce. Aujourd'hui les villages du Pogon albanais ne comptent plus que 648 habitants (données provisoires du recensement de 2000) contre 5165 en 1990 ! Dans la vallée du Drinos et du Ksérias, les villages du Dropull, zone montagneuse et sèche, se sont vidés aux deux tiers. Seules les localités proches de Gjirokastër conservent un certain dynamisme. Mais, dans ces villages pluricommunautaires, la plupart des "minoritaires" sont partis grâce aux facilités offertes par les autorités grecques et ceux qui sont restés (mis à part les gros entrepreneurs de Frashtani) se sont transformés en travailleurs frontaliers partant chaque jour à Ioannina ou y résidant pour ne rentrer dans leur village qu'en fin de semaine. Les différents investisseurs de la zone qui ont choisi de mettre à profit la proximité de la frontière, sont même contraints de faire venir de la main d'œuvre des villages avoisinants ou de Gjirokastër.

Les Valaques sont aussi partis en grand nombre. Dans le village de Mingull en Lunxhëri, où ils constituaient l'immense majorité des habitants (66\% des 105 personnes recensées dans le village en 1990) ils sont nettement plus partis (plus de $70 \%$ d'entre eux contre $46 \%$ pour les autres habitants du village). Ils se sont dirigés majoritairement vers Athènes, Pyrgos, Volos et Sparte, destinations que l'on a retrouvées pour d'autres Valaques ayant transité par Képhalovrisso (destinations qui semblent bien correspondre à l'espace migratoire du village dans les décennies précédentes). Dans les autres villages de Lunxhëri, ces anciens déclassés ont même été les acteurs d'une migration réussie et ils sont jalousés des autres habitants car ils ont pu se faire construire de belles demeures et ont parfois même ouvert des petits commerces ou acheté un mini-bus 44 .

\section{LIEUX SAS OU RÉSEAUX-SAS ?}

Les réseaux préexistants sont donc à l'origine de pratiques spatiales particulières puisqu'ils donnent à certains lieux de la frontière le rôle de relais, d'étape. À partir de ces points les migrants suivent des réseaux anciens pour chercher du travail et un endroit pour s'établir. Ils trouvent ainsi soutien et assistance dans leurs premières démarches. Mais tout comme le passage dans le village-étape de la frontière n'a qu'un temps, les migrants qui nous ont dé-

44 Ces transports collectifs privés, bien connus sous le nom de furgon, peuvent sillonner toute l'Albanie, assurant les transports aussi bien locaux que nationaux et ayant pour avantage la rapidité sur les transports en commun plus traditionnels comme les bus ou les rares trains. 
crit leur parcours ne semblent pas demeurer éternellement dans le réseau qui les a propulsés avec aisance en Grèce. En effet, il apparaît plus comme un passage, un instrument pour réaliser le projet migratoire. Une fois les conditions réunies, l'immigré s'en éloigne pour voler de ses propres ailes.

\section{ILLUSTRATION 3}

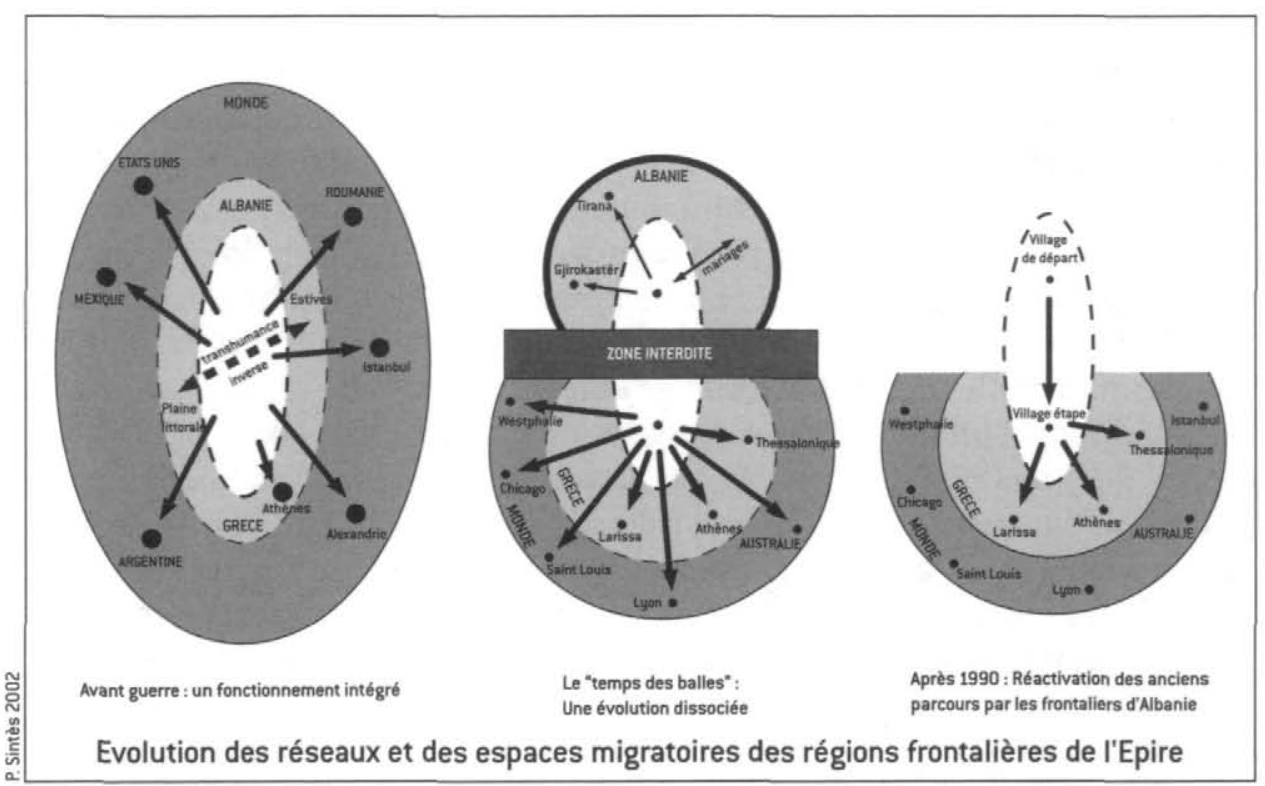

Ioannis est un Valaque de Nokov, village de Lunxhëri. Il est parti par Sopik en 1991 et s'est rendu directement à Képhalovrisso. Après un moment passé là, il s'est rendu compte qu'il ne trouverait pas de travail dans la région et est parti à Larissa. Il y avait la-bas son oncle maternel - qui est un Grec de Képhalovrisso. Au début, il est resté trois mois dans la maison de son oncle puis, celui-ci lui a trouvé un autre logement. Ses frères et le reste de sa famille l'ont suivi un an après. Au bout de deux années, tous ses frères sont partis à Athènes et lui est allé à Volos (où se trouvent de nombreuses autres personnes ayant transité par Képhalovrisso) avec sa femme et ses enfants.

Dans le domaine du fonctionnement migratoire, identifier les intermédiaires ou les différents sas par lesquels passe le migrant permet de comprendre les ressorts de son comportement. On a eu coutume de considérer, pour des migrations non-encadrées "Sud / Nord" auxquelles est comparable la migration des Albanais vers la Grèce, que les espaces urbains du pays d'émigration peuvent jouer un rôle intermédiaire dans le départ. Dans le cas des Albanais, les villes - qu'il s'agisse de la capitale, Tirana, ou même des centres régionaux - sont complètement absentes des récits de voyage des migrants 
ayant quitté le sud du pays. Pourtant, les sas sont nécessaires pour entrer dans le fonctionnement du pays d'accueil. Ils constituent un lieu d'acclimatation et de prise de repères. On a constaté que, pour les Albanais, la campagne grecque avait pu jouer ce rôle. En effet, nombre des personnes rencontrées ont commencé par travailler dans les zones agricoles avant de venir en ville.

Pour ce qui est des Albanais partis de la région frontalière que nous avons présentés ci-dessus, les réseaux préexistants à la migration ont permis d'assurer considérablement les premiers pas en Grèce. Pour eux, ce n'est pas un simple lieu qui aurait servi de sas. Le premier village traversé où ils pouvaient retrouver leur famille apparaît comme une étape plus ou moins confortable et obligée. En revanche, c'est l'ensemble des liens qui ont été renoués à partir de cette visite qui agissent comme le véritable sas pour ces migrants. En cela, le territoire de leur réseau familial leur sert de premier cercle protecteur dans un univers inconnu.

Cette première mobilité est propre aux Albanais de la frontière. Elle suit des logiques qui leur sont particulières et n'ont rien à voir avec les logiques de l'espace grec proprement dites. En ce sens, on peut les ranger dans la catégorie de mobilités inverses aux logiques de l'espace grec. En revanche, une fois acclimatés aux fonctionnements du pays d'accueil, ils peuvent s'éloigner de leurs bienfaiteurs pour regagner des lieux plus dynamiques (dans le cas de Ioannis et ses frères : Athènes et Volos) se conformant ainsi aux différences de potentiel propres à l'espace du pays d'accueil. Ils deviennent alors les acteurs de mobilités conformes.

Donc, plus qu'un lieu, c'est un réseau de relations, un territoire discontinu, qui a servi dans ce cas de sas migratoire. Convoquer les relations familiales n'a rien d'étonnant dans le cas des migrations balkaniques. Les solidarités groupales ont, en effet souvent vertébré les mouvements de population dans la région comme le décrit déjà J. Cvijić au début du siècle.

\section{CONCLUSION}

L'étude du cas de la frontière épirote n'a certes pas permis d'aborder l'ensemble des solidarités qui avaient été évoquées en première partie. Elle a néanmoins été l'occasion d'examiner le fonctionnement de réseaux transfrontaliers efficaces. On a pu ainsi établir que ces réseaux influaient grandement sur le cours de la migration. Sur sa forme dans un premier temps : la migration est plus encadrée et passe par des canaux originaux avec des étapes particulières ; mais aussi sur son calibre puisque - facilitant les démarches administratives, la recherche d'un logement ou d'un emploi - c'est un plus grand nombre de personnes qui a pu migrer. Ces réseaux ont un rôle précis dans l'histoire migratoire : ils sont utilisés comme un premier recours par le migrant, comme un sas protecteur faisant transition entre deux conditions. Leur emploi n'est donc 
pas exclusif et les migrants se montrent capables les dépasser, une fois leur pertinence épuisée, pour se fondre dans d'autres logiques de mobilités.

Il apparaît impossible de postuler que les résultats seraient les mêmes si l'on se penchait sur le cas des Tchams ou, encore moins, des Arvanites qui n'ont, a priori, aucune relation véritable avec les Albanais d'aujourd'hui. Mais, à travers ces exemples, c'est l'intervention de solidarités connexes qui retient l'attention comme un élément supplémentaire de compréhension de la structure de la migration des Albanais vers la Grèce. Dans cette perspective, tant sont noueux les chemins de l'identité45, que des solidarités recréées sont tout à fait plausibles, même pour des groupes lointains et qu'elles peuvent jouer un rôle sur les mécanismes migratoires. Mais ce type de solidarités ne pourrait en aucun cas engendrer de véritables réseaux comparables à ceux que nous avons décrits plus haut ${ }^{46}$. Le fonctionnement de réseaux préexistants n'a été observé qu'à partir des communautés de la frontière. Leur impact dépasse néanmoins cette zone étroite car, en raison des mobilités des uns et des autres depuis une cinquante d'années, ces réseaux peuvent aujourd'hui lier deux points situés n'importe où sur le territoire des deux pays.

Si ce texte avait pour point de départ la confrontation entre l'analyse de l'échelle globale et un travail de terrain détaillé, il a aussi permis une approche plus complète du comportement migratoire en lui-même. Le cas des frontaliers a été l'occasion de mettre en avant l'emploi de deux logiques territoriales : l'une aréolaire conforme aux réalités spatiales de l'économie du pays d'accueil, l'autre réticulaire autour des liens nés de la reconnaissance identitaire. Les récits de vie ont montré néanmoins que ces deux logiques étaient loin de s'exclure l'une l'autre au point même qu'elles laissent cours à une forme de va-etvient entre elles, paradoxe apparent qui revient à établir une équivalence structurelle entre deux ordres pour le moins contrastés : l'un pragmatique, massif et indiscutable - qui relève des répartitions économiques - et l'autre subjectif et discontinu, - l'espace relationnel des solidarités, l'“extraterritorial" pour reprendre le terme propre aux fonctionnements diasporiques décrits par E. Ma Mung47. A l'échelle individuelle, le migrant concilie ces deux formes territoriales opposées, les emprunte au gré de son histoire dans la mesure où elles sont, l'une comme l'autre, chargées de ressources potentielles lui permettant de faciliter son installation dans le pays d'accueil.

45 À cet égard, les débats apparus dans les années 1990 sur l'origine des Arvanites et l'opposition entre les

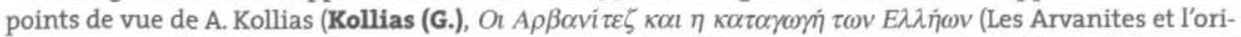

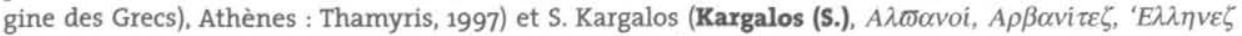
(Albanais, Arvanites, Grecs), Athènes : Sideris, 2000 [1999]) sont révélateurs.

$4^{6} \mathrm{La}$ distance est en effet bien grande entre les uns et les autres même si la reconnaissance d'une proximité linguistique peut permettre des formes d'entraides ponctuelles observées sur le terrain.

47 Ma Mung (Emmanuel), La diaspora chinoise, géographie d'une migration, Gap : Géophrys, 2000. 